\section{Die Welt aus den Angeln heben} Zur Geschichte des Climate Engineering

von Gerhard Sardemann, ITAS

Ein knapper Abriss der Geschichte des Climate Engineering wird in diesem Beitrag geliefert. Er handelt im Wesentlichen von der Entwicklung des Wunsches des Menschen, seine Umwelt zu kontrollieren und zu verbessern. Der Abriss beginnt bei den frühen Versuchen des Regenmachens und reicht von den Ideen, die Oberfläche der Erde durch den Bau von Dämmen und Kanälen zu verändern und Meeresströmungen umzulenken bis hin zu Eingriffen in die Zirkulation der Atmosphäre, die insbesondere nach der Einführung von Computern und Computermodellen möglich schienen. Climate Engineering als „technological fix" für ungewollte Beeinträchtigungen des Klimas und der Atmosphäre durch die auf Technik basierende Entwicklung des Menschen wird seit Mitte der 1960er Jahre in den USA und der Sowjetunion diskutiert, ist in diesem Sinne aber noch nie realisiert worden. Am Anfang stand die Beeinflussung der Sonneneinstrahlung, während das Management des Kohlenstoffkreislaufes erst in den 1970er Jahren in Erwägung gezogen wurde.

\section{Einleitung}

Die Verwendung der Begriffe „Climate Engineering“ und „Geoengineering“" ist erst seit Anfang der 1990er Jahre gebräuchlich. Den Begriff des „Geoengineering“ im Zusammenhang mit dem Klimaproblem gibt es aber schon seit 1977, als er von Marchetti quasi en passant im Titel seiner Arbeit über das Verklappen von $\mathrm{CO}_{2}$ am Meeresboden eingeführt wurde. Davor, und zwar seit etwa 1945, spricht man in amerikanischen Publikationen von Weather- oder Climate-,,Control“ bzw. „Modification“. Dabei bedeutet Control von vorneherein eine beabsichtigte Beeinflussung, während es bei Modification, insbesondere wenn es sich um das Klima handelt, auch um eine unbeabsichtigte Beeinflussung durch den Menschen gehen kann - eben eine, die durch die ,anthropogene" Freisetzung von $\mathrm{CO}_{2}$, Luftschadstoffen, Staub oder Wärme hervorgerufen wird. Außer in den USA beschäftigte man sich auch in der damaligen Sowjetunion mit der Verbesserung (улучшение) oder Beeinflussung (воздействие) des Klimas, wobei die Entwicklung dort der amerikanischen zum Teil voranging. Auf die sowjetischen Beiträge wurde man im Westen erst durch die amerikanischen Übersetzungen und Bibliographien aufmerksam, die im Übrigen vor allem deswegen angefertigt wurden, um über den Gegner im Kalten Krieg Bescheid zu wissen.

Der geschichtlichen Entwicklung des Geoengineering hat sich insbesondere James Rodger Fleming als ausgewiesener „Klimahistoriker“ mit einer Zahl kritischer Arbeiten angenommen, in denen er die militärische Vergangenheit des Geoengineering betont (Fleming 1998, ders. 2004, ders. 2006, ders. 2007). Auch Spencer Weart gibt in seinem im Internet offen zugänglichen Werk „The Discovery of Global Warming" interessante Einblicke in die Geschichte des Geoengineering (Weart 2009).

Keith hat in einem Übersichtsartikel den Begriff Geoengineering als eine (a) mit Vorsatz durchgeführte (b) großskalige Manipulation der Umwelt definiert und als weitere Bedingung hinzugefügt, dass es sich dabei (c) um eine Gegenmaßnahme oder einen "technological fix“ handele (Keith 2000). Mit dem Begriff „Climate Engineering" wird das Thema weiter eingegrenzt auf das Problem des Klimawandels, manchmal soll das aber auch nur heißen, dass man sich mit dem „Strahlungsmanagement“ (SRM) befasst und nicht mit der Entfernung von Kohlendioxid aus der Atmosphäre (CDR). Wigley (2006) unterscheidet in diesem Sinne zwischen Climate Engineering und Carbon Cycle Engineering.

Bei der Beschäftigung mit den Wurzeln des Climate Engineering wird man sich nicht auf Aktivitäten beschränken können, für die alle drei Faktoren der Keith'schen Definition zutreffen. So trifft z. B. auf das „Regenmachen“ (oder „-verhindern“) nur der, Vorsatz' zu. Aber gerade die in den 1950er Jahren durchgeführten Versuche des „cloud seeding”, ob zu Zwecken der Bewässerung, im kriegerischen Einsatz oder zur Abwehr von Hurrikanen, zeigen schon deutlich, mit welchen grundsätzlichen Schwierigkeiten man auch beim Climate Engineering zu rechnen haben wird. Das reicht vom Nachweis der Wirksamkeit des eingesetzten Verfahrens über 
die vermuteten Nebenfolgen bis hin zu ethischen und (völker-)rechtlichen Gesichtspunkten. Andere Vorschläge zur Klimamodifikation, darunter auch solche, die man heutzutage durchaus dem Climate Engineering zurechnet, sind ursprünglich nicht als Lösung eines Problems vorgebracht worden, sondern sollten zunächst nur der Verbesserung und Optimierung des Klimas dienen.

\section{Der Glaube versetzt Berge: Wetterschießen und Pluvikultur}

Beginnen wir mit etwas, das mit Climate Engineering nur die Absicht zur Manipulation gemein hat: das Wetter zu verändern, Unwetter zu verhindern oder Trockenheiten zu beenden. Auf den einschlägigen Volks- oder Aberglauben sei hier nur kurz verwiesen. So wurde für jede Art von Klimastress ein besonderer Heiliger angerufen; was so weit geht, dass es möglich ist, die Zahl von Bittprozessionen und die dabei angebeteten Schutzheiligen als Proxy für das vergangene Klima heranzuziehen (Pfister 1999, S. 17). Gegen drohende Unwetter, insbesondere Hagel, ging man mit viel Getöse vor und veranstaltete mit Böllern ein Wetterschießen im Glauben, damit die Wolken auseinandertreiben zu können. 1750 ließ Kaiserin Maria Theresia das Wetterschießen in ihren Landen verbieten, es soll sich aber bis heute regional gehalten haben.

Auch die ersten praktischen Versuche zur Regenerzeugung in der zweiten Hälfte des 19. Jahrhunderts waren mit viel Lärm verbunden. Nachdem schon Plutarch darüber berichtete, dass es nach großen Schlachten besonders intensiv regne und man dies nach der Einführung des Schießpulvers durchaus bestätigt fand, wurden 1890 in den USA öffentlich geförderte Tests mit Sprengstoff gemacht, der von Ballonen und Raketen in die Atmosphäre gebracht und dort gezündet wurde. Die Förderung der Versuche wurde aber nach drei Jahren wieder eingestellt, nachdem vor allem Meteorologen Zweifel an der Wissenschaftlichkeit des Verfahrens vorbrachten (Byers 1974).

Einen der ersten Vorschläge auf wissenschaftlicher Basis, bei dem es um die Erzeugung von Regen ging, machte James P. Espy im Jahr 1839: Das Entzünden großer Feuer würde demnach zu lokalen Aufwinden und der Entstehung von Cumulus-Wolken mit Niederschlag führen. Erst 115 Jahre später hat man Feldversuche in Frankreich und in Afrika durchgeführt (Byers 1974). Übrigens sah Espy die Atmosphäre damals schon als gigantische Wärmekraftmaschine. In seiner ,pathological history of weather and climate modification" beginnt für J.R. Fleming mit Espy der erste der drei Zyklen von „Promise“ und „Hype“ (Fleming 2006). Dieser erste Zyklus, der ungefähr 100 Jahre andauerte, war in den USA vor allem gekennzeichnet durch die Unternehmungen der ,pluviculturists“, die mit allerlei pseudo-wissenschaftlich verbrämtem Mummenschanz die Erzeugung von Regen versprachen.

\section{Die Welt aus den Angeln heben?}

Den Prototyp des modernen technikgetriebenen Geoengineering findet man bei Jules Verne in seinem Roman „Sans Dessus Dessous“ von 1889. Dieser Roman, auf den auch Fleming (2006) hinweist, handelt von dem Versuch, die Erdachse durch das Abfeuern eines entsprechend massiven Projektils geradezustellen, dadurch das arktische Eis abzuschmelzen und der Welt ein gleichmäBigeres Klima ohne die lästigen Jahreszeiten zu bescheren. Hauptgrund des irrwitzigen Unterfangens war allerdings der Abbau der unter dem Eis vermuteten riesigen Kohlevorräte. Was die mathematischen Grundlagen für das Verfahren angeht, so hat sich Jules Verne für diesen Roman eine wissenschaftliche Vorlage mit dem Titel „Le Titan moderne" von dem französischen Minenbauingenieur Albert Badoureau ausarbeiten lassen. ${ }^{1}$ $\mathrm{Zu}$ den erwarteten Nebenfolgen durch das Geradestellen der Erdachse gehörten dramatische Änderungen des Meeresspiegels und Verne spricht von unvorstellbaren Wanderungsbewegungen der Erdbewohner. Aufgrund eines Rechenfehlers verschwand das von einer Riesenkanone im Kilimandscharo abgefeuerte Projektil glücklicherweise wirkungslos im Weltall. Noch im Jahr 1956 nahm der amerikanische Vizepräsidentschaftskandidat Estes Kefauver dieses Szenario zum Anlass, nach der Wirkung einer Wasserstoffbombenexplosion auf die Lage der Erdachse zu fragen. Man konnte ihn jedoch beruhigen und verwies auf die stabilisierende Wirkung des Äquatorwulstes der 
Erde, was im Übrigen auch schon Jules Verne und Badoureau getan hatten (MacDonald 1969).

Durchaus einer Realisierung näher waren Ideen, die tiefer liegenden Teile der Sahara unter Wasser zu setzen, um dort blühende Landschaften zu schaffen. Jules Verne verewigte sie 1905 in seinem Roman „L'invasion de la mer“. Schon viele Jahre früher, in der Pariser Zeitschrift „La Revue Moderne“ vom November 1869, schlägt Georges Lavigne zu diesem Zweck den Bau eines Kanals vor, der viel kürzer sein könne als der soeben feierlich eröffnete Suez-Kanal. Die Sahara sei ein Krebs, der an Afrika nage, unheilbar, und man könne sich seiner nur entledigen, indem man ihn ertränke (nach Marçot 2004). Ob die Idee nun von Lavigne stammte oder dem Geographen Francois Élie Rodaire, jedenfalls machte sich letzterer mit Unterstützung von Ferdinand de Lesseps an die Verwirklichung des Projekts, das nach van Laaks (1999) letztendlich an ,allzu großer Zögerlichkeit" scheiterte und sicherlich auch daran, dass sich de Lesseps mit dem Bau des Panama-Kanals übernommen hatte. Das endgültige Aus kam 1892, aber die Idee selbst wurde nicht zu den Akten gelegt und noch etwa 20 Jahre später ist ein Prof. Etchegoyen damit in Paris erneut aufgetreten (Thompson 1912).

Das Projekt ist eines der vielen „Weißen Elefanten", die Dirk van Laak in seinem gleichnamigen Buch über den Anspruch und das Scheitern technischer Großprojekte im 20. Jahrhundert anführt (van Laak 1999). Ein anderes war der gigantische „Atlantropa-Plan“ des deutschen Architekten Herman Sörgel aus dem Jahr 1927. Durch einen Damm vor der Straße von Gibraltar sollte das Mittelmeer von der „Frischwasser"-Zufuhr des Atlantiks abgetrennt werden und somit durch den Verlust an Wasser aufgrund der vergleichsweise hohen Verdunstung an Volumen und Oberfläche abnehmen; dass dabei das untergegangene Atlantis wieder auftauchen würde, hat dem Unternehmen den Namen gegeben. Sörgel verstand sein Projekt vor allem als Mittel zur Zusammenarbeit aller maßgeblichen Völker Europas. „Die zunehmende Kooperation auf allen Gebieten hätte eine langfristige Friedensgarantie bedeutet" (van Laak 1999, S. 170). Sörgel hatte aber auch keine Skrupel, sein Projekt den Nationalsozialisten anzudie- nen und hat die Realisierung Atlantropas bis zu seinem Tod 1952 betrieben.

Im Grunde schon moderner und weniger statisch als diese Vorschläge, die an Wasserspiele am Strand erinnern, waren die Pläne Thomas Jeffersons, die man in seinen 1786 veröffentlichten gesammelten Papieren nachlesen kann. Er wollte den Isthmus von Panama öffnen, zunächst durch einen kleinen Kanal, um danach die Kräfte der Natur walten zu lassen, die durch Strömungen eine größere Öffnung erzeugen würden. Durch diese Maßnahme wäre vor allem der Schifffahrt gedient, insbesondere durch die Eliminierung des Golfstroms, der das Navigieren damals so schwer machte (vgl. Fleming 1998, S. 30). Jefferson dachte dabei noch nicht an die Auswirkungen des Golfstroms auf das Klima, aber spätestens Anfang des 20. Jahrhunderts gab es Vorschläge, den Golfstrom umzulenken, um zu einem gleichmäßigeren Klima zu kommen. Jedenfalls wettert 1929 H.A. Marmer in der Geographical Review gegen ,the absurdity of the proposals for changing the course of the Gulf Stream in the interests of a more equable climate". (Marmer 1929, S. 476) ${ }^{2}$

In der Sowjetunion dachte man noch in den 1960er Jahren daran, die Beringstraße durch einen Staudamm abzuriegeln, dort ein riesiges Atomkraftwerk zu bauen, um eine Art künstlichen Golfstrom zur Erwärmung des Nordpolarmeeres zu erzeugen (Borisov 1969; van Laak 1999). Dieses und andere in diesen Jahren vorgeschlagene Großprojekte hatten letztlich das Ziel, das Eis des Polarmeeres abzuschmelzen und das Klima in den Polarregionen gemäßigter zu gestalten. Man sah sich hier vom Programm der Kommunistischen Partei angespornt, den Reichtum und die Kräfte der Natur im Interesse des Volkes zu nutzen und dafür auch Methoden zur Beeinflussung des Klimas zu entwickeln (Rusin, Flit 1964).

\section{Nun machen wir uns unser Wetter selbst: Modellieren und Modifizieren}

Die im Folgenden zitierte Literatur aus der Zeit nach dem 2. Weltkrieg bescherte dem Autor eine Art Déjà-vu-Erlebnis. Schließlich war sie zu einem großen Teil schon zur Abfassung eines Buchbeitrages zur historischen Entwicklung der Wahrnehmung des $\mathrm{CO}_{2}$-Problems herangezogen 
worden (Sardemann 1997), ohne aber dabei zu registrieren, dass neben der unbeabsichtigten Beeinflussung und Beeinträchtigung der Atmosphäre durch Landnutzungsänderungen und die Freisetzung von Staub und Abgasen fast immer auch deren absichtliche Beeinflussung bis hin zu einer Kontrolle des Wetters und des Klimas mitgedacht wurde. Diese Kontrollmöglichkeiten wurden einerseits als große Chance gesehen, die Umwelt des Menschen zu sichern und zu verbessern, vor allem aber ging es darum, im Kalten Krieg den Gegner beeindrucken zu können.

Mit dem Aufkommen elektronischer Rechner zum Ende des Zweiten Weltkrieges steht die exakte Erfassung des Wettergeschehens und die sichere Wetterprognose als Menetekel am Himmel. Allerdings waren es keine Meteorologen, die 1945 diese Hoffnungen schürten und in Gedanken sogar noch einen Schritt weiter gingen: „Even the farmer benefits little by weather prediction; it would not seem sufficient to tell him, however accurately, that he will starve in the coming season. In weather control meteorology has a new goal worthy of the greatest effort." (Zworykin 1945, S. 78) Es soll nun aber nicht der Faden wieder aufgenommen werden, der sich von der „Pluvikultur“ weiterspinnt über den immensen Entwicklungsaufwand zur lokalen Wetterbeeinflussung in den 1950er oder 1960er Jahren, dem Einsatz während des Vietnamkrieges, bis hin zu aktuellen Anwendungen beispielsweise zur Kontrolle des Wetters während der Olympischen Sommerspiele 2008 in China.

Hier geht es um die 1945 in Princeton in den USA eingeleitete Entwicklung hin zu einer numerischen Modellierung des Wettergeschehens, der großräumigen Zirkulation der Atmosphäre und letztendlich des Klimas - mit dem Hintergedanken, hierdurch auch die Folgen eines Eingriffs in das Wettergeschehen oder Klima abschätzbar zu machen. Die Initiatoren dieser Entwicklung waren, wie gesagt, keine Meteorologen, sondern es handelte sich um den Fernsehpionier russischer Abstammung Vladimir Kosma Zworykin, damals Associate Research Director des Radio Corporation of America (RCA) Laboratory in Princeton und den in Budapest geborenen Mathematiker John von Neuman, der am Manhattan Projekt mitgearbeitet hatte und am Institute for Advanced
Study ebenfalls in Princeton wirkte. Es ist das Verdienst von James R. Fleming, das fast vergessene Papier von Zworykin vom Oktober 1945 mit dem Titel „Outline of Weather Proposal“ (1945), zusammen mit Kommentaren von J. v. Neumann und dem aus Südafrika stammenden Ozeanographen Athelstan F. Spilhaus, ausgegraben und in der von ihm herausgegebenen Zeitschrift „History of Meteorology" veröffentlicht zu haben. ${ }^{3}$

In diesem Outline preist Zworykin die Vorteile einer verlässlichen Wettervorhersage, die angesichts der Entwicklung neuartiger elektronischer Rechenanlagen und einem wesentlich erweiterten meteorologischen Messnetz und moderner Übertragungstechnologien in den Rahmen der Möglichkeiten gerückt sei. Er fügt hinzu: ,... weather prediction based on a scientific knowledge of the factors influencing weather would be the first step in any attempt in the control of weather, a goal recognized as eventually possible by all foresighted man." (Zworykin 1945, S. 61). Nach Zworykin waren alle bisherigen Vorschläge, das Wettergeschehen lokal oder global (letzteres bspw. durch das Umlenken des Golfstroms oder anderer Meeresströmungen) zu beeinflussen durch einen Mangel an ,adequate knowledge of cause and effect" gekennzeichnet (ders. 1945, S. 65). Allerdings gilt das auch für die von ihm selber vorgetragenen Vorschläge. So schlägt er, den Krieg wohl noch vor Augen, den großflächigen Einsatz von Flammenwerfern oder die Freisetzung atomarer Energie vor. Die Strahlungsbzw. Wärmebilanz ließe sich durch künstlichen Nebel, durch Öl auf dem Wasser oder Aluminium oder Ruß (,carbon“) auf Landflächen beeinflussen. Meeresströmungen könnten durch Wärmequellen oder -senken, physikalische Barrieren oder aber Veränderung der Oberflächenbeschaffenheit beeinflusst werden. Geographische Veränderungen (z. B. durch die großräumige Beeinflussung der Vegetation) hätten ebenfalls weitreichende Klimaänderungen zur Folge. Zworykin räumt ein, dass eine experimentelle Überprüfung der genannten Vorschläge äußerst schwierig sei und verweist auf die Möglichkeiten, stattdessen ein „rapidly computing model“ einzusetzen (ders. 1945, S. 66). Abschließend findet sich in seinem Outline der Vorschlag zur Schaffung einer internationalen Organisation, in deren Aufga- 
benbereich es einerseits fallen würde, den Schaden durch „,catastrophic disturbances“ abzuwenden und andererseits ,to benefit the world to the greatest extent by improved climatic conditions where possible." Er fügt unter Betonung hinzu: „Such an international organization may contribute to world peace by integrating the world interest in a common problem and turning scientific energy to peaceful persuits." (ders. 1945, S. 68).

Von Neumann hat das Thema mit Zworykin diskutiert und war überzeugt, dass das Problem der Wettervorhersage eine ideale Anwendung für die neu entwickelten Elektronengehirne wäre, auf denen gerade die Berechnungen zur Wasserstoffbombe liefen. Das Thema Wetter- und Klimabeeinflussung greift er übrigens auch in seinem häufig zitierten Beitrag „Can we survive Technology?“ 1955 in Fortune auf, wo er allerdings auch auf gar nicht so friedfertige Wettermodifikationen hinweist. Er setzt hier die globalen Folgen aus dem Einsatz von Atomwaffen mit den ebenso globalen Folgen von Klimamanipulationen auf eine Stufe (von Neumann 1955, S. 151).

\section{Einen Mangel beheben}

Die Behebung eines vom Menschen verursachten Schadens war im Jahr 1965 das Thema des Berichts „Restoring the Quality of our Environment", der vom "Science Advisory Committee“ (PSAC) des damaligen US-Präsidenten Johnson erarbeitet worden war. Um die negativen Auswirkungen eines Anstiegs des $\mathrm{CO}_{2}$-Gehalts in der Atmosphäre zu kompensieren, wurden von der betreffenden PSAC-Arbeitsgruppe um Roger Revelle Geoengineering-Maßnahmen vorgeschlagen. Die Reduktion anthropogener Emissionen oder Entfernung von $\mathrm{CO}_{2}$ aus der Atmosphäre wurde nicht in Erwägung gezogen, stattdessen ging es um eine Erhöhung der Erdalbedo beispielsweise durch das Ausbringen von reflektiven Partikeln auf den Ozeanen. Für eine einprozentige Erhöhung der Albedo wurden pro Jahr 500 Millionen Dollar an Kosten veranschlagt (PSAC 1965).

Im März des Jahres 1969 fasst Joseph O. Fletcher, Polarflieger, Geophysiker und Mitarbeiter der „RAND Corp.“, seine Gedanken zum Thema „Managing Climatic Ressources“ in einem internen Papier zusammen, worin er sich häufig auch auf aktuelle Arbeiten sowjetischer Autoren bezieht. Er weist darin auf die Notwendigkeit hin, unbeabsichtigten Einflüssen der Menschen auf die Atmosphäre entgegenzuwirken, wobei im Kontext seiner weiteren Ausführungen davon auszugehen ist, dass dabei an eine Kompensation durch eine ingenieurtechnische Beeinflussung der Atmosphäre und des Klimas gedacht wird: „The inadvertent influences of man's activity may eventually lead to catastrophic influences on global climate unless ways can be developed to compensate for undesired effects. Whether the time remaining for bringing this problem under control is a few decades or a century is still an open question." (Fletcher 1969, S. 15). Fletcher gebraucht in seinem Text übrigens den Begriff "climate machine", was ja den Zugriff durch den Ingenieur durchaus nahelegt, wohingegen der Doyen unter den deutsche Klimatologen, Hermann Flohn, betonte, dass man die Diskussion von Projekten zur Klimabeeinflussung, die damals auch in Deutschland aufgrund der aus den USA herüberschwappenden Nachrichten im Gespräch waren, nicht nur Journalisten oder Ingenieuren überlassen sollte, „deren hochfliegende Phantasie in keiner Weise durch nüchternes Fachwissen des Meteorologen eingeschränkt wird“ (Flohn 1970, S. 161). Im selben Aufsatz Flohns findet sich folgendes Zitat, das die Ziele der Wetterkontrolle mit denen des im heutigen Sinne gebrauchten Geoengineering zur Behebung der durch menschliche Beeinflussung verursachten Schäden zu versöhnen scheint (wobei man über den grammatikalischen Fehler im Original hinweglesen möge): „Früher oder später muss unter dem Druck des fortschreitenden Bevölkerungswachstums die unbeabsichtigte Klimamodifikation in eine geplante, durch Modellrechnungen in ihren Effekten vorausberechnete Klimaverbesserungen übergehen" (Flohn 1970, S. 163).

In jenen Jahren ist es allerdings gar nicht so sehr eine $\mathrm{CO}_{2}$-induzierte Klimaerwärmung, gegen die man die Methoden der Klimakontrolle in Stellung bringen wollte, schließlich sah man sich sinkenden Temperaturen entgegen, sondern fürchtete auf der einen Seite ganz allgemein die „Grenzen des Wachstums" und beobachtete andererseits in vielen Regionen der Welt humani- 
täre Katastrophen, die durch die Unbilden des Klimas verursacht schienen.

\section{Kohlenstoff-Management}

Im Laufe der 1970er Jahre schwindet der Glaube an die Möglichkeiten, die Folgen des anthropogenen Einflusses auf das Klima durch gewollte Klimabeeinflussung zu kompensieren. So wird im NAS-Bericht aus dem Jahre 1977 mit dem Titel „Energy and Climate” der Vorschlag des PSAC aus dem Jahr 1965, die Albedo der Erde durch das Verteilen reflektiver Partikel auf den Ozeanen zu erhöhen, wegen seiner Nebenwirkungen als undurchführbar angesehen: „The disadvantages of such a scheme are obvious and may be insuperable. The material might eventually pile up on the coastlines of the world, with unacceptable environmental consequences, and the effect on fisheries might be disastrous." (NAS 1977, S. 13) Stattdessen werden Maßnahmen erwogen, das $\mathrm{CO}_{2}$ in Biomasse zu überführen und dort $\mathrm{zu}$ speichern. Zum einen denkt man daran, die Senkenfunktion des Ozeans zu vergrößern, indem durch Düngung die photosynthetische Produktion organischer Kleinstlebewesen erhöht wird. Durch den Einsatz von 10 Mio. Tonnen Phosphor könnten 300 Mio. Tonnen an organischem Kohlenstoff durch Absinken aus den oberen Wasserschichten entfernt werden. Außerdem geht es um eine Vergrößerung des Baumbestandes in den bestehenden Waldgebieten der Erde, wobei die Entwicklung durch Wirtschafts- und Bevölkerungswachstum eher in die entgegengesetzte Richtung, nämlich Verringerung der Waldflächen gehe, oder aber auch um die Anlage von Plantagen, deren Holz man nach dem Schlagen gegen Verfall schützen müsse, um das $\mathrm{CO}_{2}$ dauerhaft zu binden. Letzteres hatte 1976 schon Freeman J. Dyson vorgeschlagen (Dyson 1976). Andererseits sollte es aber auch möglich sein, Biomasse energetisch einzusetzen, um damit fossile Brennstoffe einzusparen. Die dadurch zu erreichende Vermeidung fossiler $\mathrm{CO}_{2}$-Emissionen wird dabei als der vielversprechendere Weg angesehen.

Über den Umgang mit $\mathrm{CO}_{2}$ aus einer recht allgemeinen, systemanalytischen Perspektive machte man sich schon um 1975 im International Institute for Applied Systems Analysis im österreichischen Laxenburg (IIASA) verstärkt Gedanken, wo das „Energy Systems Program“ unter Wolfgang Häfele Wissenschaftler aus aller Welt anzog. So schrieb William D. Nordhaus seine Ideen zu diesem Thema, die er zusammen mit Cesare Marchetti ausgearbeitet hatte, in einem internen IIASA-Bericht nieder (Nordhaus 1975). Ausgehend von einer Betrachtung des Kohlenstoffkreislaufes und möglicher anderer Kontrollstrategien, die er in der unten im Original reproduzierten, etwas launigen Tabelle 1 zusammenfasste, geht es Nordhaus um die Frage: ,... how can we limit the concentration of atmospheric carbon dioxide to a reasonable level?" (Nordhaus 1975, S. 9) Wenn er in der Tabelle von „band-aids“ (Pflaster) spricht, dürfte er damit die im Text genannte Verhüllung arktischen Eises mittels ,gauze“ (Gaze; aber auch: Verbandmull) zur Veränderung der Albedo gemeint haben.

\section{Tab. 1: Kontrollstrategien ${ }^{4}$}

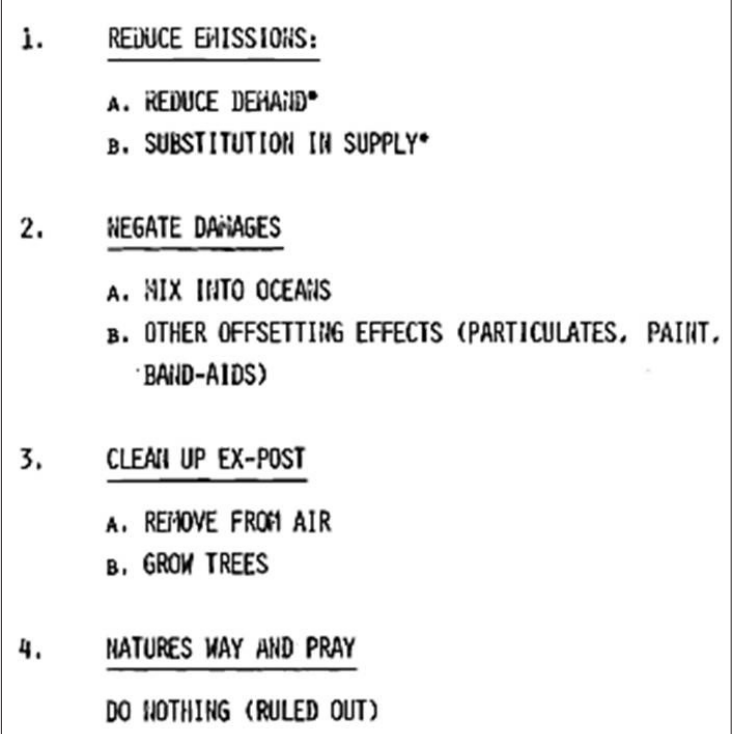

Quelle: Nordhaus 1975, S. 7

Marchetti schlug damals, ebenfalls in einem internen IIASA-Bericht (mit der Nummer RM76-17, 1976), zum ersten Mal vor, $\mathrm{CO}_{2}$ aus dem Abgasstrom von Kraftwerken abzutrennen, zu verflüssigen und in der Tiefsee zu deponieren. Im darauffolgenden Jahr erschien dieser Bericht als Veröffentlichung in der ersten Ausgabe der gerade neu von Stephen H. Schneider gegründeten Zeitschrift „Climatic Change“. Im Titel des 
Berichts „On Geoengineering and the $\mathrm{CO}_{2}$ Problem“ (Marchetti 1977) taucht zum ersten Mal das Wort „Geoengineering“ im Zusammenhang mit der Lösung des $\mathrm{CO}_{2}$-Problems auf. Marchetti hält es hier nicht mehr für nötig, diesen Begriff im Text einzuführen, im zugrundeliegenden IIASA-Bericht heißt es aber zumindest im Vorwort, Geoegineering sei ,a kind of ,system synthesis" where solutions to global problems are attempted from a global view".

Es scheint aber, dass dieser Begriff in den folgenden Jahren ohne Widerhall bleibt, bevor er 1992 im Jahr der Verabschiedung der Klimarahmenkonvention im NAS-Bericht „Policy Implications of Greenhouse Warming: Mitigation, Adaptation, and the Science Base" wieder aufgegriffen und als Oberbegriff für nahezu alle heute noch diskutierten Ansätze des Climate Engineering eingeführt wird. Dabei wurde (fast möchte man hinzufügen: „natürlich“) nicht vergessen, darauf hinzuweisen, dass ,... because the climate system and its chemistry are poorly understood, these options must be considered extremely carefully." (NAS 1992, S. 59) Als dann 1994 auf dem „AAAS Annual Meeting“ in San Francisco die Frage gestellt wird „Could We/Should We Engineer the Earth"s Climate?", liegt die Benutzung des Begriffs „Climate Engineering“ natürlich nicht fern. Der einzige Beitrag, der zumindest in der Ankündigung der Veranstaltung den Begriff „Climate Engineering“ in der Überschrift trägt, stammt übrigens aus dem Los Alamos National Laboratory und enthält den verräterischen Satz: „Many Climate Engineering needs match those of defense technologies" (Canavan 1994).

\section{Schlussfolgerungen}

Es ist charakteristisch für Climate Engineering, dass mit der Verbrennung fossiler Brennstoffe zwar ein Experiment globalen Ausmaßes begonnen wurde, dass aber alle Vorschläge, die jemals eine Klimamodifikation in einer ähnlichen Größenordnung zum Ziel hatten, von einer Realisierung weit entfernt waren und immer noch sind. Es handelt sich hier also vornehmlich um eine Geschichte von mehr oder weniger plausiblen Theorien und Ideen sowie von Erfindungen, die bislang keiner ausprobieren konnte.
Die Vorstellung vom Wetter und Klima als etwas den Gesetzen der Mechanik Unterworfenem, als Wärmekraftmaschine, mathematischem oder Computer-Modell führte zu der Überzeugung, man brauche nur an den richtigen Stellschrauben zu drehen, um zum gewünschten Ergebnis zu kommen. In den meisten Fällen geht es um eine Verbesserung oder Optimierung der Umwelt des Menschen. Bei den Großprojekten, die Ende des 19. und Anfang des 20. Jahrhunderts vorgeschlagen wurden, stand die Idee von einem großen Ganzen im Vordergrund, während beim modernen Geoengineering oder Climate Engineering ganz partikulär die Problemlösung der treibende Faktor ist.

Viele halten Geo- oder Climate Engineering schon allein deshalb für diskreditiert, weil es sich bei den Ideengebern häufig um Ingenieure und um Militärs handelt. Immer wieder stößt man auch in der neueren Zeit auf Namen, die man eher mit der Entwicklung der Atom- und Wasserstoffbombe verbindet als mit Klimaproblemen. Die Anfangszeiten waren zudem von Abenteurern, Scharlatanen und spleenigen Einzelkämpfern bevölkert. Auch wenn von allen Seiten immer wieder betont wurde und auch immer noch wird, dass die Kontrolle des Wetters und Klimas nur in internationaler Zusammenarbeit möglich sein sollte, zeigt ein Blick in die Vergangenheit, dass immer unilateral und meist mit militärischem Hintergrund agiert wurde. So dürfte Geoengineering auch heute noch eine militärische Option für den Fall sein, dass durch Klimaänderungen induzierte Konflikte überhand nehmen sollten.

Ein Blick in die Vergangenheit zeigt auch, dass immer wieder Ideen bei aus heutiger Sicht fundamentalem Unwissen in die Welt gesetzt wurden. Auf der einen Seite waren dies Menschen mit großer Fantasie, aber kaum Fachwissen, denen auf der anderen Seite Fachleute gegenüberstanden (darunter Meteorologen und Meereskundler), die zur Zurückhaltung mahnten. Viele Ideen sind aufgrund ihrer „Nebenwirkungen“ verworfen worden - teils aufgrund neuerer Forschungsergebnisse, teils aufgrund widersprüchlicher Ergebnisse bei Feldexperimenten, wenn sie denn durchgeführt wurden. Oder es setzte sich der gesunde Menschenverstand durch, nachdem der erste „hype“ der Idee vorüber war. 
Die hier dargestellten Positionen zur Geschichte des Climate Engineering sind nur eine erste Skizze und der Bias zu Gunsten der US-amerikanischen Literatur ist augenscheinlich. Insbesondere bei der sowjetischen und russischen Literatur, aber auch im nicht-angelsächsischen Sprachraum wäre genauer zu recherchieren. Wenn wir allerdings heute im ,westlichen Wissenschaftssystem“ von Climate Engineering sprechen, bezieht sich das im Wesentlichen auf Entwicklungen in den USA, auch wenn viele der ursprünglichen Ideen aus der Sowjetunion kamen. Dass man dort, bzw. heute in Russland, ebenfalls, ja fast noch stärker und vielleicht unbekümmerter, die Tradition der Wetter- und Klimamodifikation aus der Mitte des letzten Jahrhunderts weiterpflegt, zeigt beispielsweise der aktuelle Aufsatz von Izrael et al. (2009), der das Potenzial unterschiedlicher Geoenginee-

\section{Einschlägige Begriffe und Abkürzungen in diesem Schwerpunkt zu Climate Engineering}

\begin{tabular}{|c|c|c|}
\hline$C E$ & Climate Engineering & $\begin{array}{l}\text { Eine technikorientierte Strategie, um den beschleunigten Klimawandel im } \\
\text { Notfall ingenieurwissenschaftlich zu mildern oder zu bekämpfen. }\end{array}$ \\
\hline$S R M$ & Solar Radiation Management & $\begin{array}{l}\text { CE durch Beeinflussung der kurzwelligen Strahlungsbilanz der Erdatmo- } \\
\text { sphäre a) durch Abschattung mittels Folien und Spiegeln im All oder b) } \\
\text { durch eine Veränderung der Albedo der Erde }\end{array}$ \\
\hline$C D R$ & Carbon Dioxide Removal & $\begin{array}{l}\text { CE-Konzept mit dem Ziel, } \mathrm{CO}_{2} \text { als Ursache für den Klimawandel langfri- } \\
\text { stig aus der Atmosphäre zu entfernen. Letztendlich geht es dabei um eine } \\
\text { Modifikation der langwelligen Strahlungsbilanz bzw. deren Rückgängig- } \\
\text { machung. }\end{array}$ \\
\hline$C C S$ & Carbon Capture and Storage & $\begin{array}{l}\text { Abscheiden von } \mathrm{CO}_{2} \text { direkt aus dem Abgasstrom mit dem Ziel es mög- } \\
\text { lichst dauerhaft unterirdisch zu speichern. Erste Vorschläge sahen eine } \\
\text { Lagerung auf dem Meeresgrund vor. CCS zählt zu den CDR-Konzepten. }\end{array}$ \\
\hline$I P C C$ & $\begin{array}{l}\text { Intergovernmental Panel on } \\
\text { Climate Change }\end{array}$ & $\begin{array}{l}\text { Der „Zwischenstaatlicher Ausschuss für Klimaänderungen“ fasst in re- } \\
\text { gelmäßigen Abständen den Wissensstand der Klimaforschung zusammen. } \\
\text { Der letzte ausführliche Bericht (AR4) erschien } 2007\end{array}$ \\
\hline UNFCCC & $\begin{array}{l}\text { United Nations Framework } \\
\text { Convention on Climate Change }\end{array}$ & $\begin{array}{l}\text { Klimarahmenkonvention der Vereinten Nationen; in Kraft getreten am } 21 . \\
\text { März } 1994\end{array}$ \\
\hline$K P$ & Kyoto Protokoll & $\begin{array}{l}\text { Protokoll zur Klimarahmenkonvention, in dem zum ersten Mal verbind- } \\
\text { liche Reduktionsverpflichtungen für die Industrieländer (außer den USA) } \\
\text { festgelegt wurden. Das } 1997 \text { in Kyoto ausgehandelte und } 2005 \text { in Kraft } \\
\text { getretene Protokoll läuft im Jahr } 2012 \text { aus. }\end{array}$ \\
\hline$C D M$ & Clean Development Mechanism & $\begin{array}{l}\text { Einer der projektbezogenen Mechanismen des KP, der die nachhaltige } \\
\text { Entwicklung in den Entwicklungs- und Schwellenländern fördern soll und } \\
\text { es den Industrieländern erlaubt, sich in solchen Projekten erzielte Emissi- } \\
\text { onsreduktionen gutschreiben zu lassen }\end{array}$ \\
\hline$J I$ & Joint Implementation & $\begin{array}{l}\text { Ein weiterer der projektbezogenen Mechanismen des KP zur gemeinsa- } \\
\text { men Umsetzung von Projekten in den Industrieländern }\end{array}$ \\
\hline$a$ & Albedo & $\begin{array}{l}\text { Maß für das Rückstrahlvermögen von Oberflächen ( } \mathrm{a}=0 \text { bei voller } \mathrm{Ab}- \\
\text { sorption; } \mathrm{a}=1 \text { bei voller Reflektion) Die kurzwellige Albedo bezieht sich } \\
\text { auf die Sonneneinstrahlung und erreicht bei Schnee und Wolken einen } \\
\text { Wert von a }=0,9 \text {, während Wasser und Wald mit Albedowerten bis } 0,05 \\
\text { dunkel erscheinen. }\end{array}$ \\
\hline & Tipping Points & $\begin{array}{l}\text { Beim Überschreiten der sog. „Kipp-Punkte“ wechselt das Klima unauf- } \\
\text { haltsam in einen anderen stabilen Zustand. Der Übergang ist möglicher- } \\
\text { weise mit abrupten, drastischen Klimaänderungen verknüpft und der } \\
\text { resultierende Klimazustand könnte es in manchen Regionen recht unge- } \\
\text { mütlich werden lassen. }\end{array}$ \\
\hline
\end{tabular}

Quelle: Eigene Zusammenstellung / GS 
ring-Ansätze miteinander vergleicht. Darin wird zwar, gerade wenn es um aktuelle Arbeiten geht, auf Quellen im Westen verwiesen, aber u. a. auch an den Vorschlag des russischen Raumfahrtpioniers Ziolkovski aus den 1920er Jahren erinnert, im All reflektierende Objekte zu stationieren, um die Erde vor der Sonnenstrahlung abzuschirmen. In der im Westen quasi „Geoengineering-freien Phase" in den 1980ern hinterließ der Auftritt von Budyko 1988 auf dem Weltkongress „Climate and Development" in Hamburg ein zwiespältiges Echo, als er vor der Limitierung des Einsatzes fossiler Brennstoffe (,carbon fuels“) warnte und betonte, dass es doch möglich sei, aktiv auf das Klima einzuwirken, falls es notwendig werden sollte, die globale Erwärmung zu stoppen (Budyko, Sedunov 1988).

\section{Anmerkungen}

1) Siehe dazu Dehs 2005, S. 262.

2) Schon 1924 hatte der deutsche Meereskundler Gerhard Schott von der „Torheit von Vorschlägen (...) zur Verbesserung des Klimas" gesprochen und nannte als Beispiele die Absicht, „die Belle-IsleStraße zu sperren, um dem eisführenden Labradorstrom den Zugang zum Golf von St. Lorenz zu verwehren“ oder im Falle Wladiwostoks ,die kalte Strömung, die an der Westseite des Japanischen Meeres zieht, von dem Eintritte in den Hafen abzuhalten" (Schott 1924, S. 151).

3) Von Athelstan F. Spilhaus stammt auch das oben angeführte Zitat über die vom Hunger bedrohten Farmer.

4) Die Tabelle wurde nicht ins Deutsche übertragen, da es dem Autor um den Tenor der englischen Forumilierung geht (die Redaktion).

\section{Literatur}

Borisov, P.M., 1969: Can We Control the Arctic Climate? In: Bulletin of the Atomic Scientists 25/3 1969, S. 43-48

Budyko, M.I.; Sedunov, Yu.S., 1988: Anthropogenic Climatic Changes. Prepared for the World Congress Climate and Development, 7.-10. November 1988, Congress Centrum Hamburg

Byers, H.R., 1974: History of Weather Modification. In: Hess, W.N. (ed.): Weather and Climate Modification. New York

Canavan, G.H., 1994: Space Sensors for Global Change. Presented at the American Association for the Advancement of Science Annual Meeting San Francisco, California. 19-23 February 1994, Los Alamos National Laboratory

Dehs, V., 2005: Jules Verne. Eine kritische Biographie. Artemis und Winkler. Düsseldorf

Dyson, F.J., 1976: Can We Control the Carbon Dioxide in the Atmosphere? In: Energy 2 (1976), S. 287-291

Fleming, J.R., 1998: Historical Perspectives on Climate Change. New York

Fleming, J.R., 2004: Fixing the Weather and Climate: Military and Civilian Schemes for Cloud Seeding and Climate Engineering. In: Rosner, L. (ed.): The Technological Fix. How people use technology to create and solve problems. New York, S. 175-200

Fleming, J.R., 2006: The pathological history of weather and climate modification: Three cycles of promise and hype. In: Historical Studies in the Physical and Biological Sciences 37/1 (2006), S. 3-25

Fleming, J.R., 2007: The Climate Engineers. In: Wilson Quarterly 31/2 (2007), S. 46-60

Fletcher, J.O., 1969: Managing Climatic Resources. The RAND Corporation. Santa Monica, CA, USA

Flohn, H., 1970: Produzieren wir unser eigenes Klima? In: Meteorologische Rundschau 21/6 (1970), S. 161-164

Izrael, Yu.A.; Ryaboshapko, A.G.; Petrov, N.N., 2009: Comparative Analysis of Geo-engineering Approaches to Climate Stabilization. In: Russian Meteorology and Hydrology 34/6 (2009), S. 335-347

Keith, D.W., 2000: Geoengineering the Climate: History and Prospect. In: Annual Review of Energy and the Environment 25 (2000), S. 245-284

MacDonald, G.J.F., 1969: Wie man die Welt zerstören kann. In: Calder, N. (Hg.): Eskalation der neuen Waffen. München, S. 189-210

Marçot, J.-L., 2004: Appel d'imaginaire: La mer intérieure africaine 1869-1887. http://jeanlouis.marcot. free.fr/meresume.htm (download 9.7.10)

Marchetti, C., 1977: On Geoengineering and the $\mathrm{CO}_{2}$ Problem. In: Climatic Change 1/1 (1977), S. 59-68

Marmer, H.A., 1929: The Gulf Stream and Its Problems. In: Geographical Review 19/3 (1929), S. $457-478$

NAS - National Academy of Sciences, 1977: Energy and Climate: Studies in Geophysics. Washington, DC NAS - National Academy of Sciences, 1992: Policy Implications of Greenhouse Warming: Mitigation, Adaptation, and the Science Base. Washington, DC

Nordhaus, W.D., 1975: Can We Control Carbon Dioxide? IIASA Working Paper WP-75-63 
PSAC - President's Science Advisory Committee, 1965: Restoring the Quality of Our Environment. Washington, DC

Pfister, Chr., 1999: Wetternachhersage. 500 Jahre Klimavariationen und Naturkatastrophen (14961995). Bern

Rusin N.P.; Flit, L.A., 1964: Man Versus Climate. U.S. Department of Commerce, Office of Technical Services, Joint Publications Research Service, Washington, DC

Sardemann, G., 1997: Beeinflussung des globalen Klimas durch den Menschen: Historische Entwicklung und Stand des Wissens zum anthropogenen Treibhauseffekt. In: Kopfmüller, J.; Coenen, R. (Hg.): Risiko Klima. Der Treibhauseffekt als Herausforderung für Wissenschaft und Politik. Frankfurt, S. 27-73 (Veröffentlichungen des Instituts für Technikfolgenabschätzung und Systemanalyse (ITAS), Bd. 4) Schott, G., 1924: Physische Meereskunde. Berlin

Thompson, G.A., 1912: A Plan for Converting the Sahara Desert into a Sea. In: Scientific American August 10, S. 114-125

van Laak, D., 1999: Weiße Elefanten. Anspruch und Scheitern technischer Großprojekte im 20. Jahrhundert. Stuttgart

von Neumann, J., 1955: Can We Survive Technology? In: Fortune, June, S. 106-108, S. 151-152

Weart, S., 2009: The Discovery of Global Warming. Juli 2009 Version. http://www.aip.org/history/ climate/ (download 9.7.10)

Wigley, T.M.L., 2006: A Combined Mitigation/Geoengineering Approach to Climate Stabilization. In: Science 314 (2006), S. 452-454

Zworykin, V.K., 1945: Outline of Weather Proposal, October 1945. Princeton, NJ: RCA Laboratories, 12 p. + appendices. In: History of Meteorology 4 (2008), S. $57-78$

\section{Kontakt}

Dipl.-Meteorologe Gerhard Sardemann

Karlsruher Institut für Technologie (KIT)

Institut für Technikfolgenabschätzung und Systemanalyse (ITAS)

Postfach 36 40, 76021 Karlsruhe

Tel.: +49 (0) 7247 / 82 - 2734

Fax: +49 (0) 7247 / 82 - 4806

E-Mail: gerhard.sardemann@kit.edu

\section{Climate Engineering zwischen Klimapolitik und Völkerrecht} Status quo und Perspektiven

\author{
von Thilo Wiertz und David Reichwein, Uni- \\ versität Heidelberg
}

In den letzten Jahrzehnten haben sich eine Reihe von Prinzipien in der internationalen Umwelt- und Klimapolitik etabliert. Anhand existierender Mechanismen und völkerrechtlicher Verträge diskutiert der Beitrag, welche Implikationen diese für den Umgang mit Climate Engineering haben. Die Techniken könnten insofern zur Bewährungsprobe für die derzeitige Klimapolitik werden, als ihr Einsatz keinen internationalen Konsens erfordert. Zentral für die politische Diskussion von Climate Engineering wird daher der Aspekt der intertemporalen und geographischen Risikoverteilung sein. Ob und inwieweit das Völkerrecht Einsatz und Erforschung von Climate Engineering betrifft, hängt wesentlich von der Interpretation bestehender Verträge $a b$.

\section{Einleitung}

Die technologische Möglichkeit einer globalen Klimamanipulation wirft die Frage auf, wie über einen Einsatz von Climate Engineering entschieden werden sollte. Dabei geht es um mehr als den ,richtigen” Zielwert einer globalen Oberflächentemperatur, wie es naturwissenschaftliche Betrachtungen bisweilen nahelegen: Climate Engineering bewegt sich in einem Spannungsfeld von Risiken, die dem Klimawandel einerseits und einer gezielten Klimaveränderung andererseits zugeschrieben werden. Angesichts räumlich differenzierter Auswirkungen und der Kontextualität von Wertmaßstäben sind so sehr unterschiedliche Vorstellungen davon möglich, wann und in welchem Maße technische Eingriffe in das globale Klimasystem sinnvoll oder gar notwendig sind. Die politische Herausforderung ist es also zu klären, in welchen institutionellen Zuständigkeitsbereichen eine Entscheidung über Climate Engineering verortet wird und welchen moralischen Rechtfertigungen sie folgt. Bislang existieren zwar kaum internationale Rahmenbedingungen, die sich explizit mit Climate En- 